\title{
Study for Reaching a Degradation Test Plan
}

\author{
Tzong-Ru Tsai ${ }^{\mathrm{a}}$, Y. L. Lio ${ }^{\mathrm{b}}$ \\ ${ }^{a}$ Department of Statistics, Tamkang University, Taiwan \\ ${ }^{b}$ Department of Mathematical Sciences, University of South Dakota, USA \\ *Corresponding Author: trtsai@stat.tku.edu.tw
}

\begin{abstract}
In this paper, we study the merits and drawbacks of using the algorithm proposed by Tsai et al. ${ }^{(1)}$ to obtain optimal sample size allocation and termination times of a twovariable constant-stress accelerated degradation test plan under the stochastic process of Gamma. A simulation example of light emitting diodes is used for illustrating the implementation of the algorithm.
\end{abstract}

Keywords: Brownian motion process, Gamma process, geometric Brownian motion process, generalized Eyring model, inverse Gaussian distribution.

\section{Introduction}

It is very difficult to obtain the failure times of highly reliable products via using traditional life test methods, such as the censoring test, truncated test or accelerated life test. To overcome this difficulty, accelerated degradation test methods can be used to replace these traditional life test methods. In practice, the degradation of a surrogate variable, which is highly related to the lifetime, can be used to infer the lifetime distribution parameters, and the obtained degradation paths are modeled by stochastic models. Brownian motion (BM) and geometric Brownian motion (GBM) stochastic processes have been widely used to model the degradation of a product over time. Even if the BM and GBM processes are easy to apply through the use of the assumption of normal-distributed increments of degradation (or damage) within consecutive measure times at an original scale and logarithmic transformation, respectively. However, the assumption of normal-distributed increments is not true for some realistic cases because the increments of a degradation (or damage) process from a BM or GBM could be positive (or negative) (see ${ }^{(1)}$ ). A Gamma process (GP) always exhibits a monotone-increasing pattern and is more reasonable to model a damage process for performing reliability assessment than $\mathrm{BM}$ or GBM, see ${ }^{(1)-(11)}$. The Gamma process stands for the Gamma degradation process.

In this paper, we consider the constant-stress accelerated degradation test (CSADT) method to obtain the degradation information for statistical inference. To minimize the variance of the mean time to failure (MTTF) subject to budget, Tsai et al. ${ }^{(1)}$ provided an inference method to develop the optimization system. Because it is not easy to reach the sampling plan of the sample size allocation and termination times of the CSADT, an algorithm was provided by Tsai et al. ${ }^{(1)}$ to implement their proposed method. We would like to study the merits and drawbacks of the computational $\operatorname{algorithm}^{(1)}$.

A lot of studies have been provided to discuss the onevariable CSADT method and optimal design. When two stress variables are used for a CSADT, the parameter estimation and optimal design become complicated. Because many modern products are highly reliable, using two stress variables are better than using one stress variable to accelerate the degradation of products under a degradation test. Two-variable CSADT method can help practitioners to obtain more degradation information for reliability analysis within the affordable testing time. Comprehensive discussions about using different accelerated degradation methods can be found in works ${ }^{(12)-(32)}$.

The remainder of this paper is organized as follows: The inference method of Tsai et al. ${ }^{(1)}$ is addressed in Section 2. In Section 3, the algorithm to reach an optimal design plan of the sample size allocation and termination times is given. Moreover, the merits and drawbacks of using this algorithm are studied. Simulation method to obtain a GP is discussed and a simulated example is used to illustrate the application of the algorithm. Finally, the conclusions are given in Section 4. 


\section{The inference method}

Based on the working assumption $\mathrm{A} 4^{(1)}$ : Let the starting time of the ADT $t_{i j 0}=0$ and the initial damage of each unit used for life testing $x_{i j 0}=0$. The damage of each surviving unit at $L_{i}=\left(L_{1 i}, L_{2 i}\right)$ is measured at times $t_{i j 1}<t_{i j 2}<\cdots<t_{i j m_{i}}$ and labeled by $x_{i j 1}, x_{i j 2}, \ldots, x_{i j m_{i}}$, respectively. The increment of the damage process, $y_{i j h}=$ $x_{i j h}-x_{i j(h-1)}$, follows a Gamma distribution with a shape coefficient $v \tau_{i j h}$ and a scale parameter $\beta$, where $\tau_{i j h}=$ $t_{i j h}-t_{i j(h-1)}$ for $j=1,2, \ldots, q_{i}, \quad h=1,2, \ldots, m_{i}$ and $i=1,2, \ldots, k$. Linking the shape coefficient with two stress variables $L_{1 i}$ and $L_{2 i}$ through using the generalized Eyring model (GEM):

$$
\begin{gathered}
v_{L_{i}}=\exp \left(\gamma_{0}+\gamma_{1} L_{1 i}+\gamma_{2} L_{2 i}+\gamma_{3} L_{1 i} L_{2 i}\right), i= \\
1,2, \ldots, k .
\end{gathered}
$$

The GEM model is a generalized form that includes three widely used single-loading acceleration models - the Arrhenius law model, a power law model and an exponential law model - as special cases when only one of stress variable is not considered. Let $S$ denote the first passage time of the GP to the threshold $C . S$ follows an Inverse Gaussian distribution, which was defined by the following probability density function, based on the approximation procedure of [4]:

$$
g_{S}(s ; C)=g_{S}\left(s ; x_{0}=0, C\right)=\frac{c_{\beta} / \sqrt{v}}{\sqrt{2 \pi s^{3}}} \exp \left[-\frac{v\left(s-C_{\beta} / v\right)^{2}}{2 s}\right] .
$$

The log-likelihood function can be presented by:

$$
\begin{aligned}
& \ell(\Theta) \propto \sum_{i=1}^{k} \sum_{l=1}^{p_{i}} \log \left(g_{S}\left(s_{i l} ; C\right)\right)+ \\
& \sum_{i=1}^{k} \sum_{j=1}^{q_{i}} \sum_{h=1}^{m_{i}} \log \left(f_{A}\left(y_{i j h} ; \tau_{i j h}\right)\right)
\end{aligned}
$$

wher $\Theta=\left(\gamma_{0}, \gamma_{1}, \gamma_{2}, \gamma_{3}, \beta\right)$, and $f_{A}\left(\because \tau_{i j h}\right)$ is the probability density function of the degradation measurements at time $\tau_{i j h}$ for the modeling of $f_{A}\left(\because ; \tau_{i j h}\right)$ $\left(\sec ^{(4)}\right)$. The maximum likelihood estimates $\hat{\gamma}_{0}, \hat{\gamma}_{1}, \hat{\gamma}_{2}, \hat{\gamma}_{3}$ and $\hat{\beta}$ are the solutions to maximizing $\ell(\Theta)$ in (2). The approximate MTTF formula can be obtained by $\left(\mathrm{see}^{(4)}\right)$ :

$$
\mu_{L_{0}} \simeq \frac{C_{\beta}}{v_{0}}+\frac{1}{2 v_{0}}=\frac{1}{\xi}\left(\frac{C}{\beta}+\frac{1}{2}\right),
$$

where the asymptotic variance of $\hat{\mu}_{L_{0}}$ can be obtained by

$$
\operatorname{AVar}\left(\hat{\mu}_{L_{0}}\right)=h^{T} \mathbf{I}^{-1}(\Theta) h
$$

and $h^{T}=\left[\partial \mu_{L_{0}} / \partial \gamma_{0}, 0,0,0, \partial \mu_{L_{0}} / \partial \beta\right]=[-C / \beta \xi-1 /$ $\left.2 \xi, 0,0,0,-C / \beta^{2} \xi\right] . \quad \mathbf{I}^{-1}(\Theta)$ is the inverse of Fisher information matrix provided by ${ }^{(1)}$.

The total cost (TC) based on sample size vector $\mathbf{n}=$ $\left(n_{1}, n_{2}, \ldots, n_{k}\right)$ and the termination time vector $\mathbf{t}=$ $\left(t_{1}, t_{2}, \ldots, t_{k}\right)$ can be evaluated by

$$
\begin{aligned}
\mathrm{TC}(\mathbf{n}, \mathbf{t}) & =c_{0} n_{U}+c_{o p} \sum_{i=1}^{k} t_{i}+c_{L_{1}} \sum_{i=1}^{k} t_{i}\left(L_{1 i}^{\prime}-\right. \\
\left.L^{\prime}{ }_{10}\right) & +c_{L_{2}} \sum_{i=1}^{k} n_{i} t_{i} L_{2 i}^{\prime},
\end{aligned}
$$

where $c_{0}$ is the fixed cost per unit, $c_{0 p}$ is the operating cost per unit of time, $c_{L_{1}}$ is the cost to increase temperature by one degree, and $c_{L_{2}}$ is the cost to increase the current by one milliampere per unit of time for each test unit.

Let $n_{U}=\sum_{i=1}^{k} n_{i}$ denote the total sample size used in the ADT and $\delta_{i}$ be the length of the time interval between two measurements in the run $i$ of the ADT. The $\delta_{i}$ value is commonly determined according to the operation schedule of the involved laboratory. In reality, a constant measurement frequency, $\delta_{i}=\delta$, for all runs of the ADT is more convenient for practical use. The TC to implement an ADT includes the fixed cost, operating cost, and variable cost.

Based on the upper bound of the entire experimental time, and let $\operatorname{AS}(\mathbf{n}, \mathbf{t}) \equiv \operatorname{AVar}\left(\hat{\mu}_{L_{0}}\right)$. The optimal setting $\left(\mathbf{n}^{*}, \mathbf{t}^{*}\right)$ can be determined to minimizing $\operatorname{AS}(\mathbf{n}, \mathbf{t})$ subject to an upper bound of the TC, denoted by $\phi_{0}$ :

$\operatorname{Minimize} \operatorname{AS}(\mathbf{n}, \mathbf{t})$

Subject to $\mathrm{TC}(\mathbf{n}, \mathbf{t}) \leq \phi_{0}$,

$\delta \leq t_{1}, t_{2}, \ldots, t_{k} \leq t_{U}, n_{i} \geq 1, i=$

$1,2, \ldots, k$ and $\sum_{i=1}^{k} n_{i}=n_{U}$.

\section{The algorithm and example}

The optimal setting $\left(\mathbf{n}^{*}, \mathbf{t}^{*}\right)$ is difficult to obtain because the optimization system (6) is not easy to be solved. The main difficulty is that the sample sizes are discrete parameters and the termination times are continuous parameters. Moreover, there are $2 \times k$ parameters need to be solved. It is nearly impossible to achieve a feasible optimal setting $\left(\mathbf{n}^{*}, \mathbf{t}^{*}\right)$ when $k$ is large. To over such difficulties, Tsai et al. ${ }^{(1)}$ suggested to cut the termination time into discrete measures such that the optimal setting can be search in a domain of finite many points through using a global searching method. Let $t_{U}$ in (6) be presented as a multiple of $\delta$, and all products in the CSADT must be measured at least once. Following the design of Tsai et al. ${ }^{(1)}$ to obtain $\delta \leq t_{1}, t_{2}, \ldots, t_{k} \leq t_{U}$. Let

$$
B^{\prime}{ }_{t}=\left\{\mathbf{t} \mid t_{i}>0, i=1,2, \ldots, k \text { and } \mathrm{TC}(\mathbf{n}, \mathbf{t}) \leq \phi_{0}\right\} .
$$

Dividing the interval $\left[\delta, t_{U}\right]$ is divided into subintervals of equal length, and the partition points are given as $\delta, 2 \delta, \ldots, t_{U}$. Let the collection of $\left\{\delta, 2 \delta, \ldots, t_{U}\right\}$ be denoted by $B_{S}$ and

$$
B_{t}=\left\{\mathbf{t} \in B_{S} \mid \delta \leq t_{1}, t_{2}, \ldots, t_{k} \leq t_{U} \text { and } \mathrm{TC}(\mathbf{n}, \mathbf{t}) \leq \phi_{0}\right\} .
$$


The optimal CSADT plan can be established over $B_{t}$ instead of $B^{\prime}{ }_{t}$. Let $n_{B_{t}}$ be the total number of all possible combinations of $t_{1}, t_{2}, \ldots, t_{k}$ in $B_{t}$, and all these combinations are denoted by $\mathbf{t}^{(1)}, \mathbf{t}^{(2)}, \ldots, \mathbf{t}^{\left(n_{B_{t}}\right)}$.

\section{Algorithm (see $\left.{ }^{(1)}\right):$}

Step 1. Determine the set $B_{t}=\left\{\mathbf{t}^{(1)}, \mathbf{t}^{(2)}, \ldots, \mathbf{t}^{\left(n_{B_{t}}\right)}\right\}$.

Step 2. The upper bound of total sample size in the ADT at $\mathbf{t}^{(i)}$ can be evaluated by applying the condition $\mathrm{TC}(\mathbf{n}, \mathbf{t}) \leq \phi_{0}$, and be denoted by

$$
n_{U, \mathbf{t}^{(i)}}^{\prime}=\left\lfloor\frac{\psi}{c_{0}}\right\rfloor,
$$

where

$\psi=\phi_{0}-c_{o p} \sum_{i=1}^{k} t_{j}^{(i)}+c_{L_{1}} \sum_{i=1}^{k} t_{j}^{(i)}\left(L_{1 i}^{\prime}-L_{10}^{\prime}\right)+$ $c_{L_{2}} n_{L} \sum_{i=1}^{k} t_{j}^{(i)} L_{2 i}^{\prime},\lfloor x\rfloor$ is the largest integer equal or smaller than $x$, and $n_{L}$ is the smallest sample size used for each run.

Step 3. Let $n_{1}^{\prime}=\left\lfloor n_{U, \mathbf{t}^{(i)}}^{\prime} / k\right\rfloor, n_{i}^{\prime}=\left\lfloor\left(n_{U, \mathbf{t}^{(i)}}^{\prime}-\left(n_{1}^{\prime}+\right.\right.\right.$ $\left.\left.\left.\cdots+n_{i-1}^{\prime}\right)\right) /(k-i+1)\right\rfloor$ for $i=2,3, \ldots,(k-1)$ and $n_{k}^{\prime}=n_{U, t^{(i)}}^{\prime}-\left(n_{1}^{\prime}+\cdots+n_{k-1}^{\prime}\right)$.

Step 4. If TC $\left(n_{1}^{\prime}, \ldots, n_{k}^{\prime}, \mathbf{t}^{(i)}\right)<\phi_{0}$, then $n_{U, \mathbf{t}^{(i)}}=$ $n_{U_{, \prime} \mathbf{t}^{(i)}}^{\prime}$ and proceed to Step 5; otherwise, $n_{U, \mathbf{t}}^{\prime(i)}=n_{U, \mathbf{t}^{(i)}}^{\prime}-1$ and revert to Step 3.

Step 5. Replace the total sample size of the ADT at $\mathbf{t}^{(i)}$ by $n_{U, \mathbf{t}^{(i)}}$. Generate the set of all possible combinations of $\mathbf{n}_{\mathbf{t}^{(i)}}^{(j)}=\left(n_{1, \mathbf{t}^{(i)}}^{(j)}, n_{2, \mathbf{t}^{(i)}}^{(j)}, \ldots, n_{k, \mathbf{t}^{(i)}}^{(j)}\right)$ at $\mathbf{t}^{(i)}$ by

$S_{\mathbf{n}}^{(i)}$

$=\left\{\right.$ all possible $\mathbf{n}_{\mathbf{t}^{(i)}}^{(j)} \mid \sum_{i=1}^{k} n_{h, \mathbf{t}^{(i)}}^{(j)}=n_{U, \mathbf{t}^{(i)}}$ for $\left.j=1,2, \ldots, n_{S}\right\}$, where $n_{S}$ is the total number of sample size combinations such that $\sum_{i=1}^{k} n_{h, \mathbf{t}^{(i)}}^{(j)}=n_{U, \mathbf{t}^{(i)}}$.

Step 6. Compute $H\left(\mathbf{n}_{\mathbf{t}^{(i)}}^{*} \mid \mathbf{t}^{(i)}\right)$ by

$$
H\left(\mathbf{n}_{\mathbf{t}^{(i)}}^{*} \mid \mathbf{t}^{(i)}\right)=\min _{j=1,2, \ldots, n_{S}} \operatorname{AS}\left(\mathbf{n}_{\mathbf{t}^{(i)}}^{(j)}, \mathbf{t}^{(i)}\right) \text { for } i=1,2, \ldots, n_{B_{t}} .
$$

Step 7. The approximate optimal ADT plan, $\left(\mathbf{n}^{*}, \mathbf{t}^{*}\right)$, can be obtained by solving the following equation:

$$
\left(\mathbf{n}^{*}, \mathbf{t}^{*}\right)=\arg \min _{i=1,2, \ldots, n_{B}} H\left(\mathbf{n}_{\mathbf{t}^{(i)}}^{*} \mid \mathbf{t}^{(i)}\right) .
$$

The algorithm makes an optimal setting of a CSADT plan can be identified easily over searching for a finite number of combinations of parameters. Hence, we can guarantee the convergence of the algorithm. But please note that the search is over the domain $B_{t}$ but not the domain $B^{\prime}{ }_{t}$. The obtained optimal setting is only an approximate optimal solution. Moreover, the accuracy is affected by the following:

(i) The selection of $B_{t}$.

(ii) The selection of $n_{i}^{\prime}$ in Step 3 .

Luckily, for a two-variable CSADT the size of combinations of all possible combinations of $t_{1}, t_{2}, \ldots, t_{k}$ is not too large. Hence, the selection of $B_{t}$ is not a problem here. The selection of $n_{i}^{\prime}$ in Step 3 is a method to make the combinations of all possible sample sizes in the domain be easily determined. The method in Step 3 is only a feasible method for determining the sample space of the sample size allocation but not the unique method. In this optimization problem, the domain of the combinations of all possible sample sizes is a key problem and it may fail the computation. The method in Step 3 is workable, but could cause the algorithm loss some accuracy.

To generate a GP with $m$ measurements: $\left\{x_{h}, h=\right.$ $1,2, \ldots, m\}$, we can use the condition that $y_{h}=x_{h}-x_{h-1}$, follows a Gamma distribution that has shape coefficient $v_{L} \tau_{h}$ and a scale parameter $\beta$, where $\tau_{h}=t_{h}-t_{h-1}$ for $h=1,2, \ldots, m$. The link function $v_{L}=\exp \left(\gamma_{0}+\gamma_{1} L_{1}+\right.$ $\left.\gamma_{2} L_{2}+\gamma_{3} L_{1} L_{2}\right)$ can be used to generate the GP measurements through using the following steps:

Step i. Generate $m$ observations from the Gamma distribution that has shape coefficient $v_{L} \tau_{h}$ and a scale parameter $\beta$. Denote these observations by $y_{1}, y_{2}, \cdots, y_{m}$.

Step ii. Obtain $x_{1}, x_{2}, \cdots, x_{m}$ by $x_{1}=y_{1}, x_{2}=y_{2}+x_{1}=$ $y_{2}+y_{1}, x_{3}=y_{3}+x_{2}=y_{3}+y_{2}+y_{1}, \ldots, x_{m}=$ $\sum_{i=1}^{m} y_{i}$.

Using the above Step i and Step ii, the GP can be generated under the stress level combination $\left(L_{1}, L_{2}\right)$. Based on the reference parameter setting of a LED example $\left(\operatorname{see}^{(1)}\right)$, the parameters of GP can be $\gamma_{0}=-2.902, \gamma_{1}=$ $0.577, \gamma_{2}=0.533, \gamma_{3}=0.531$, and $\beta=0.662$. The stress level combinations $L_{1}^{\prime}=(40,70)$ and $L_{2}^{\prime}=(550,700)$ are considered in simulation, where $L_{1}^{\prime}$ and $L_{2}^{\prime}$ are the absolute ambient temperature in degree Celsius and drive current in milliampere. Using scale-free transformation formulas for ambient temperature and drive current, respectively, as: 


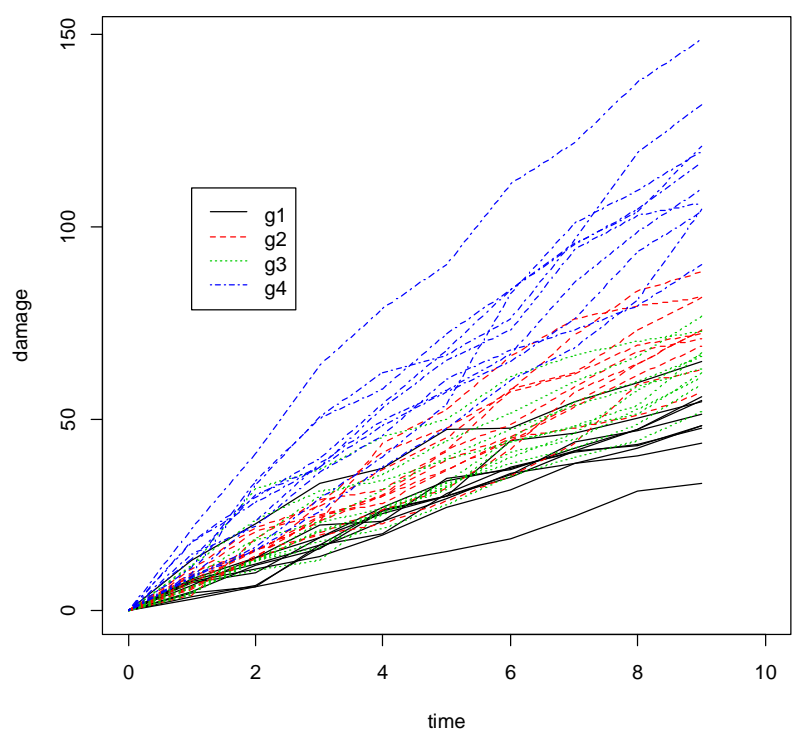

Fig. 1. The damage paths of $g 1("-"):\left(L_{1}, L_{2}\right)=(40,550)$, $g 2("---"):\left(L_{1}, L_{2}\right)=(40,700), g 3(" \ldots "):\left(L_{1}, L_{2}\right)=$ $(70,550)$ and $g 4("-\bullet-"):\left(L_{1}, L_{2}\right)=(70,700)$

Table 1. Optimal sample size allocation and termination times $\left(t_{i}\right.$ in days)

\begin{tabular}{cccccccccccc}
\hline$d_{1}$ & $\phi_{0}$ & $n_{1}^{*}$ & $n_{2}^{*}$ & $n_{3}^{*}$ & $n_{4}^{*}$ & $t_{1}^{*}$ & $t_{2}^{*}$ & $t_{3}^{*}$ & $t_{4}^{*}$ & $\mathrm{TC}$ & $\sqrt{\mathrm{AS}\left(\mathbf{n}^{*}, \mathbf{t}^{*}\right)}$ \\
\hline 5 & 8000 & 14 & 9 & 8 & 5 & 252 & 252 & 238 & 231 & 7858.33 & 116.415 \\
& 10000 & 18 & 11 & 10 & 6 & 252 & 252 & 252 & 238 & 9777.45 & 103.229 \\
& 12000 & 22 & 14 & 12 & 7 & 252 & 252 & 252 & 231 & 11727 & 93.537 \\
\cline { 2 - 10 } & 8000 & 14 & 9 & 8 & 5 & 252 & 238 & 231 & 231 & 7875.30 & 117.610 \\
& 10000 & 18 & 11 & 10 & 6 & 252 & 252 & 238 & 231 & 9780.25 & 104.043 \\
& 12000 & 22 & 14 & 12 & 7 & 252 & 252 & 238 & 231 & 11745.4 & 94.114 \\
\hline
\end{tabular}

$$
L_{1 i}=\frac{1 / L_{10}^{\prime}-1 / L_{1 i}^{\prime}}{1 / L_{10}^{\prime}-1 / L_{1 H}^{\prime}}
$$

and

$$
L_{2 i}=\frac{\log \left(L_{2 i}^{\prime}\right)-\log \left(L_{20}^{\prime}\right)}{\log \left(L_{2 H}^{\prime}\right)-\log \left(L_{20}^{\prime}\right)}, i=1,2, \ldots, k,
$$

where $L_{10}^{\prime}=25$ and $L_{20}^{\prime}=350$ are the normal use conditions, $L_{1 H}^{\prime}=70$, and $L^{\prime}{ }_{2 H}=700$. Hence, we obtain $L_{1}=(0.583,1)$ and $L_{2}=(0.652,1)$. Four groups of simulated damage paths measured in days for $n_{1}=n_{2}=$ $n_{3}=n_{4}=10$ and $m=9$ are given in Figure 1. Such kind of LEDs has a MTTF around 21000 hours, and a LED is defined as failure when the LED losses $30 \%$ or more of it luminous. In Figure 1, we found more damage for the LEDs that are subjected to higher stress loading.

\section{Conclusions}

In this study, we study the merits and drawbacks of the algorithm proposed by Tsai et al. ${ }^{(1)}$, the algorithm is used to obtain an optimal accelerated degradation test plan of the sample size allocation and termination times under the Gamma process. The algorithm, proposed by Tsai et al. ${ }^{(1)}$, is easy to be used to reach an optimal accelerated degradation test plan, but the recommended optimal accelerated degradation test plan is approximated not an exact one. Moreover, the algorithm asks the prior information of the model parameters, and such condition could be unavailable for some applications.

The implementation of using the algorithm to obtain accelerated degradation test plans is illustrated by a simulation example of light emitting diodes. We found that the recommended optimal accelerated degradation test plan would employ the total cost as close to the budget as possible. The asymptotical variance can be decreased if the more budget is allowed for the degradation test. More sample units are used for the run with lower stress loading, and fewer sample units were allocated to the run at higher stress loading.

The algorithm proposed by Tsai et al. ${ }^{(1)}$ can be used for similar optimization systems not only for the optimization system (6). The algorithm allocates the sample number into the sample space of the sample units by using a splitting method. The splitting method could be improved by other methods. How to create an optimization method such that the optimization method is free of the prior information of model parameters is also an important issue. All these two topics are interesting and will be addressed in future studies.

\section{Acknowledgment}

The authors thank the anonymous referees for their suggestions and comments that led to a considerable improvement of this article. This study is supported by the grant of Ministry of Science and Technology, Taiwan MOST 104-2221-E-032-017-MY2

\section{References}

(1) T.-R. Tsai, W.-Y. Sung, Y.L. Lio, S.-I. Chang and J.-C. Lu, "Optimal two-variable accelerated degradation test plan for Gamma degradation process", IEEE Trans. Rel. To appear. 
(2) H. Lim and B.-J. Yum, "Optimal design of accelerated degradation tests based on Wiener process models", $J$. Appl. Stat., vol. 38, no. 2, pp. 309-325, 2011.

(3) W.J. Padgett and M.A. Tomlinson, "Inference from accelerated degradation and failure data based on Gaussian process models", Lifetime Data Anal., vol. 10, no. 2, pp. 191-206, 2004.

(4) C. Park and W.J. Padgett, "Accelerated degradation models for failure based on geometric Brownian motion and gamma process", Lifetime Data Anal., vol. 11, no. 4, pp. 511-527, 2005.

(5) T.-R. Tsai, C.-W. Lin, Y.-L. Sung, P.-T. Chou, C.-L. Chen and Y.L. Lio, "Inference from lumen degradation data under Wiener diffusion process", IEEE Trans. Rel., vol. 61, no. 3, pp. 710-718, 2012.

(6) G.A. Whitmore, "Estimating degradation by a Wiener diffusion process subject to measurement error", Lifetime Data Anal., vol. 1, pp. 307-319, 1995.

(7) C.-M. Liao and S.-T Tseng, "Optimal design for stepstress accelerated degradation tests", IEEE Trans. Rel., vol. 55, no. 1, pp. 59-66, 2006.

(8) T.-R. Tsai, Y.L. Lio and N. Jiang, "Optimal decision on the accelerated degradation test plan under the Wiener process", Qual. Technol. Quant. Manag., vol. 11, no.4, pp. 461-470, 2014.

(9) S.-T. Tseng and N. Balakrishnan and C.-C. Tsai, "Optimal step-stress accelerated degradation test plan for gamma degradation process", IEEE Trans. Rel., vol. 58, no. 4, pp. 234-245, 2011.

(10) C.-C. Tsai, S.-T. Tseng and N. Balakrishnan, "Optimal burn-in policy for highly reliable products using gamma degradation process", IEEE Trans. Rel., vol. 60, no. 1, pp. 338-350, 2013.

(11) Q. Guan and Y.-C. Tang, "Optimal design of accelerated degradation test based on gamma process models", Chin. J. Appl. Probab. Statist., vol. 29, no. 2, pp. 213-224, 2013.

(12) C. Park and W.J. Padgett, "Stochastic degradation models with several accelerating variables", IEEE Trans. Rel., vol. 55, no. 2, pp. 379-390, 2006.

(13) C. Park and W.J. Padgett, "Cumulative damage models for failure with several accelerating variables", Qual. Technol. Quant. Manag., vol. 4, no. 1, pp. 17-34, 2007.

(14) J.M. Van Noorwijk, "A survey of the application of gamma processes in maintenance", Rel. Eng. Syst. Safety, vol. 94, no. 1, pp. 2-21, 2009.

(15) J.Y. Peng, "A note on optimal allocations for the second elementary symmetric function with applications for optimal reliability design”, Nav. Res. Logis., vol. 59, no. 3-4, pp. 278-284, 2012.

(16) J.L. Folks and R.S. Chhikara, "The inverse Gaussian distribution and its statistical application-A review", $J$. Roy. Statist. Soc., ser. B, vol. 40, no. 3, pp. 263-289, 1978.

(17) Y. Xiang, D.W. Coit and Q. Feng, "Accelerated burn-in and condition-based maintenance for $n$-subpopulations subject to stochastic degradation", IIE Trans., vol. 46, no.10, pp. 1093-1106, 2014.

(18) C.-J. Lu and W.Q. Meeker, "Using degradation measures to estimate a time-to-failure distribution", Technometrics, vol. 35, no.2, pp. 161-174, 1993.

(19) H.T. Liao and E.A. Elsayed, "Reliability prediction and testing plan based on an accelerated degradation rate model", Int. J. Mater. Prod. Technol., vol. 21, no.5, pp. 402-422, 2004.

(20) C. Park and W.J. Padgett, "Cumulative damage models based on gamma processes, In F. Ruggeri, F. Faltin, and R. Kenett, editors", Enc. Statist. Qual. Rel., 1-5, John Wiley \& Sons: New York.

(21) S.-T. Tseng and C.-Y. Peng, "Stochastic diffusion modeling of degradation data", J. Data Sci., vol. 5, pp. 315-313, 2007.

(22)Z. Pan, and N. Balakrishnan, "Reliability modeling of degradation of products with multiple performance characteristics based on gamma processes", Rel. Eng. Syst. Safety, vol. 96, pp. 949-957, 2011.

(23) Z.-S. Ye, Y. Wang, K.-L. Tsui and M. Pecht, "Degradation data analysis using Weiner process with measurement errors", IEEE Trans. Rel., vol. 62, no. 4, pp. 772-780, 2013.

(24) G. Jin and D. Matthews, "Reliability demonstration for long-life products based on degradation testing and a Wiener process model", IEEE Trans. Rel., vol. 63, no.3, pp. 781-797, 2014.

(25) S.-T. Tseng, N. Balakrishnan and C.-C. Tsai, "Optimal step-stress accelerated degradation test plan for gamma distribution process", IEEE Trans. Rel., vol. 58, no. 4, pp. 611-618, 2009.

(26) X. Wang and D. Xu, "An inverse Gaussian process model for degradation data", Technometrics, vol. 52 no.2, pp. 188-197, 2010.

(27)Z.-S. Ye and N. Chen, "The inverse Gaussian process as a degradation model", Technometrics, vol. 56 no.3, pp. 302$311,2014$.

(28) A. Mettas, "Modeling \& analysis for multiple stress-type accelerated life data", Proc. Annu. Reliability and Maintainability Symp., pp. 138-143, 2000. 
(29) W. Zhao and E.A. Elsayed, "A general accelerated life model for step-stress testing”, IIE Trans., vol. 37, no.11, pp. 1059-1069, 2005.

(30) L.A. Escobar and W.Q. Meeker, "A review of accelerated test models”, Statist. Sci., vol. 21, no.4, pp. 552-577, 2006.

(31) H.-Y. Xu and H.-L. Fei, "Planning step-stress accelerated life tests with two experimental variables", IEEE Trans. Rel., vol. 56, no.1, pp. 77-84, 2007.

(32) C. Li and N. Fard, "Optimal bivariate step-stress accelerated life test for censored data", IEEE Trans. Rel., vol. 56, no.3, pp. 569-579, 2007. 